International Journal of Pure and Applied Mathematics

Volume 95 No. 4 2014, 523-533

ISSN: 1311-8080 (printed version); ISSN: 1314-3395 (on-line version)

url: http://www.ijpam.eu

doi: http://dx.doi.org/10.12732/ijpam.v95i4.4

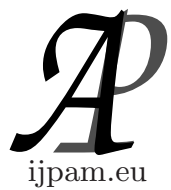

\title{
A NOVEL CRITERION FOR GUARANTEED COST CONTROL FOR UNCERTAIN SWITCHED RECURRENT \\ NEURAL NETWORKS
}

Grienggrai Rajchakit

Department of Mathematics

Maejo University

Chiangmai, 50290, THAILAND

\begin{abstract}
This paper studies the problem of guaranteed cost control for a class of uncertain switched recurrent delayed neural networks. The time delay is a continuous function belonging to a given interval, but not necessary to be differentiable. A cost function is considered as a nonlinear performance measure for the closed-loop system. The stabilizing controllers to be designed must satisfy some stability constraints on the closed-loop poles. By constructing a set of augmented Lyapunov-Krasovskii functional combined with Newton-Leibniz formula, a guaranteed cost controller is designed via memoryless state feedback control and new sufficient conditions for the existence of the guaranteed cost state-feedback for the system are given in terms of linear matrix inequalities (LMIs). A numerical examples are given to illustrate the effectiveness of the obtained result.
\end{abstract}

AMS Subject Classification: 15A09, 52A10, 74M05, 93D05, 94C10, 92B20 Key Words: uncertain switched recurrent neural networks, guaranteed cost control, stabilization, interval time-varying delays, Lyapunov function, linear matrix inequalities

\section{Introduction}

In the past few years, recurrent neural networks (RNNs) have been extensively

Received: February 6, 2014

(c) 2014 Academic Publications, Ltd. url: www.acadpubl.eu 
studied because of their applications in many areas, such as signal processing, associative memory, pattern recognition, combination optimisation and so on. In implementation of artificial neural networks, time delays are unavoidable because of finite switching speeds of the amplifiers. It is known long that delays may result in oscillation and instability (see e.g.[1-3]). So it is important to investigate the stability of delayed RNNs. Delayed RNNs have been extensively used in various engineering and scientific fields, for example, solving linear projection equations and quadratic optimisation problems [2], component detection of medical signal[3], automatic control engineering [4], analysis of moving images or speech [5], biological simulation[6] and so on. In recent years, a variety of competing sufficient stability conditions for delayed RNNs have been accumulated (see e.g.[7-9]). In many practical systems, it is desirable to design neural networks which are not only asymptotically or exponentially stable but can also guarantee an adequate level of system performance. In the area of control, signal processing, pattern recognition and image processing, delayed neural networks have many useful applications. Some of these applications require that the equilibrium points of the designed network be stable. In both biological and artificial neural systems, time delays due to integration and communication are ubiquitous and often become a source of instability. The time delays in electronic neural networks are usually time-varying, and sometimes vary violently with respect to time due to the finite switching speed of amplifiers and faults in the electrical circuitry. Guaranteed cost control problem [2, 3, 5, 7-9] has the advantage of providing an upper bound on a given system performance index and thus the system performance degradation incurred by the uncertainties or time delays is guaranteed to be less than this bound. The Lyapunov-Krasovskii functional technique has been among the popular and effective tool in the design of guaranteed cost controls for neural networks with time delay. Nevertheless, despite such diversity of results available, most existing work either assumed that the time delays are constant or differentiable [8-10]. Although, in some cases, delay-dependent guaranteed cost control for systems with time-varying delays were considered in [7,9], the approach used there can not be applied to systems with interval, non-differentiable time-varying delays. To the best of our knowledge, the guaranteed cost control and state feedback stabilization for uncertain switched recurrent neural networks with interval, non-differentiable time-varying delays have not been fully studied yet (see, e.g., [2-10] and the references therein), which are important in both theories and applications. This motivates our research.

In this paper, we investigate the guaranteed cost control for uncertain delayed switched recurrent neural networks problem. The novel features here 
are that the delayed switched recurrent neural network under consideration is with various globally Lipschitz continuous activation functions, and the timevarying delay function is interval, non-differentiable. A nonlinear cost function is considered as a performance measure for the closed-loop system. The stabilizing controllers to be designed must satisfy some exponential stability constraints on the closed-loop poles. Based on constructing a set of augmented Lyapunov-Krasovskii functional combined with Newton-Leibniz formula, new delay-dependent criteria for guaranteed cost control via memoryless feedback control is established in terms of LMIs, which allow simultaneous computation of two bounds that characterize the exponential stability rate of the solution and can be easily determined by utilizing MATLABs LMI Control Toolbox.

The outline of the paper is as follows. Section 2 presents definitions and some well-known technical propositions needed for the proof of the main result. LMI delay-dependent criteria for guaranteed cost control and a numerical examples showing the effectiveness of the result are presented in Section 3. The paper ends with conclusions and cited references.

\section{Preliminaries}

The following notation will be used in this paper. $\mathbb{R}^{+}$denotes the set of all real non-negative numbers; $\mathbb{R}^{n}$ denotes the $n$-dimensional space with the scalar product $\langle x, y\rangle$ or $x^{T} y$ of two vectors $x, y$, and the vector norm $\|.\| ; M^{n \times r}$ denotes the space of all matrices of $(n \times r)$-dimensions. $A^{T}$ denotes the transpose of matrix $A ; A$ is symmetric if $A=A^{T} ; I$ denotes the identity matrix; $\lambda(A)$ denotes the set of all eigenvalues of $A ; \lambda_{\max }(A)=\max \{\operatorname{Re} \lambda ; \lambda \in \lambda(A)\}$. $x_{t}:=\{x(t+s): s \in[-h, 0]\},\left\|x_{t}\right\|=\sup _{s \in[-h, 0]}\|x(t+s)\| ; C^{1}\left([0, t], \mathbb{R}^{n}\right)$ denotes the set of all $\mathbb{R}^{n}$-valued continuously differentiable functions on $[0, t]$; $L_{2}\left([0, t], \mathbb{R}^{m}\right)$ denotes the set of all the $\mathbb{R}^{m}$-valued square integrable functions on $[0, t]$;

Matrix $A$ is called semi-positive definite $(A \geq 0)$ if $\langle A x, x\rangle \geq 0$, for all $x \in \mathbb{R}^{n} ; A$ is positive definite $(A>0)$ if $\langle A x, x\rangle>0$ for all $x \neq 0 ; A>B$ means $A-B>0$. The notation $\operatorname{diag}\{\ldots\}$ stands for a block-diagonal matrix. The symmetric term in a matrix is denoted by $*$.

Consider the following uncertain switched recurrent neural networks with interval time-varying delay:

$$
\begin{aligned}
\dot{x}(t)= & -\left(A_{\gamma(x(t))}+\Delta A(t)\right) x(t)+\left(W_{0 \gamma(x(t))}+\Delta W_{0}(t)\right) W_{0} f(x(t)) \\
& +\left(W_{1 \gamma(x(t))}+\Delta W_{1}(t)\right) g(x(t-h(t)))+B_{\gamma(x(t))} u(t), \\
& t \geq 0, \quad x(t)=\phi(t), \quad t \in\left[-h_{1}, 0\right]
\end{aligned}
$$


where $x(t)=\left[x_{1}(t), x_{2}(t), \ldots, x_{n}(t)\right]^{T} \in \mathbb{R}^{n}$ is the state of the neural, $\gamma($.$) :$ $R^{n} \rightarrow \mathcal{N}:=\{1,2, \ldots, N\}$ is the switching rule, which is a function depending on the state at each time and will be designed. A switching function is a rule which determines a switching sequence for a given switching system. Moreover, $\gamma(x(t))=i$ implies that the system realization is chosen as the $i^{t h}$ system, $i=1,2, \ldots, N$. It is seen that the system (1) can be viewed as an autonomous switched system in which the effective subsystem changes when the state $x(t)$ hits predefined boundaries, $u(.) \in L_{2}\left([0, t], \mathbb{R}^{m}\right)$ is the control; $n$ is the number of neural, and

$$
\begin{gathered}
f(x(t))=\left[f_{1}\left(x_{1}(t)\right), f_{2}\left(x_{2}(t)\right), \ldots, f_{n}\left(x_{n}(t)\right)\right]^{T}, \\
g(x(t-h(t)))=\left[g_{1}\left(x_{1}(t-h(t))(t)\right), g_{2}\left(x_{2}(t-h(t))(t)\right), \ldots, g_{n}\left(x_{n}(t-h(t))\right]^{T},\right.
\end{gathered}
$$

are the activation functions; $A_{i}=\operatorname{diag}\left(\bar{a}_{1 i}, \bar{a}_{2 i}, \ldots, \bar{a}_{n i}\right), \bar{a}_{j i}>0, j=1,2, \ldots, n$. represents the self-feedback term; $B_{i} \in R^{n \times m}$ is control input matrix; $W_{0 i}, W_{1 i}$ denote the connection weights, the discretely delayed connection weights and the distributively delayed connection weight, respectively; the time-varying uncertain matrices $\Delta A(t), \Delta W_{0}(t)$, and $\Delta W_{1}(t)$ are defined by:

$$
\Delta A(t)=E_{a} F_{a}(t) H_{a}, \Delta W_{0}(t)=E_{w_{0}} F_{w_{0}}(t) H_{w_{0}}, \Delta W_{1}(t)=E_{w_{1}} F_{w_{1}}(t) H_{w_{1}},
$$

where $E_{a}, E_{w_{0}}, E_{w_{1}}, H_{a}, H_{w_{0}}, H_{w_{1}}$ are known constant real matrices with appropriate dimensions. $F_{a}(t), F_{w_{0}}(t), F_{w_{1}}(t)$ are unknown uncertain matrices satisfying

$$
F_{a}^{T}(t) F_{a}(t) \leq I, \quad F_{w_{0}}^{T}(t) F_{w_{0}}(t) \leq I, \quad F_{w_{1}}^{T}(t) F_{w_{1}}(t) \leq I, \quad t \in R^{+} .
$$

The time-varying delay function $h(t)$ satisfies the condition

$$
0 \leq h_{0} \leq h(t) \leq h_{1},
$$

The initial functions $\phi(t) \in C^{1}\left(\left[-h_{1}, 0\right], R^{n}\right)$, with the norm

$$
\|\phi\|=\sup _{t \in\left[-h_{1}, 0\right]} \sqrt{\|\phi(t)\|^{2}+\|\dot{\phi}(t)\|^{2}} .
$$

In this paper we consider various activation functions and assume that the activation functions $f(),. g($.$) are Lipschitz with the Lipschitz constants f_{i}, e_{i}>$ 0 :

$$
\begin{aligned}
& \left|f_{i}\left(\xi_{1}\right)-f_{i}\left(\xi_{2}\right)\right| \leq f_{i}\left|\xi_{1}-\xi_{2}\right|, \quad i=1,2, \ldots, n, \forall \xi_{1}, \xi_{2} \in \mathbb{R} \\
& \left|g_{i}\left(\xi_{1}\right)-g_{i}\left(\xi_{2}\right)\right| \leq e_{i}\left|\xi_{1}-\xi_{2}\right|, \quad i=1,2, \ldots, n, \forall \xi_{1}, \xi_{2} \in \mathbb{R}
\end{aligned}
$$


The performance index associate with the system (1) is the following function

$$
J=\int_{0}^{\infty} f^{0}(t, x(t), x(t-h(t)), u(t)) d t,
$$

where $f^{0}(t, x(t), x(t-h(t)), u(t)): R^{+} \times R^{n} \times R^{n} \times R^{m} \rightarrow R^{+}$, is a nonlinear cost function satisfies

$$
\exists Q_{1}, Q_{2}, R: f^{0}(t, x, y, u) \leq\left\langle Q_{1} x, x\right\rangle+\left\langle Q_{2} y, y\right\rangle+\langle R u, u\rangle,
$$

for all $(t, x, u) \in R^{+} \times R^{n} \times R^{m}$ and $Q_{1}, Q_{2} \in R^{n \times n}, R \in R^{m \times m}$, are given symmetric positive definite matrices. The objective of this paper is to design a memoryless state feedback controller $u(t)=K x(t)$ for system (1) and the cost function (5) such that the resulting closed-loop system

$$
\begin{aligned}
\dot{x}(t)= & -\left[\left(A_{i}+E_{a} F_{a}(t) H_{a}\right)-B_{i} K\right] x(t)+\left(W_{0 i}+E_{w_{0}} F_{w_{0}}(t) H_{w_{0}}\right) f(x(t)) \\
& +\left(W_{1 i}+E_{w_{1}} F_{w_{1}}(t) H_{w_{1}}\right) g(x(t-h(t))),
\end{aligned}
$$

is exponentially stable and the closed-loop value of the cost function (7) is minimized.

Definition 1. Given $\alpha>0$. The zero solution of closed-loop system (5) is $\alpha$-exponentially stabilize if there exist a positive number $N>0$ such that every solution $x(t, \phi)$ satisfies the following condition:

$$
\|x(t, \phi)\| \leq N e^{-\alpha t}\|\phi\|, \quad \forall t \geq 0 .
$$

Definition 2. Consider the control system (1). If there exist a memoryless state feedback control law $u^{*}(t)=K x(t)$ and a positive number $J^{*}$ such that the zero solution of the closed-loop system (7) is exponentially stable and the cost function (5) satisfies $J \leq J^{*}$, then the value $J^{*}$ is a guaranteed constant and $u^{*}(t)$ is a guaranteed cost control law of the system and its corresponding cost function.

We introduce the following technical well-known propositions, which will be used in the proof of our results.

Proposition 1. (Schur complement lemma [11]). Given constant matrices $X, Y, Z$ with appropriate dimensions satisfying $X=X^{T}, Y=Y^{T}>0$. Then $X+Z^{T} Y^{-1} Z<0$ if and only if

$$
\left(\begin{array}{cc}
X & Z^{T} \\
Z & -Y
\end{array}\right)<0
$$


Proposition 2. (Integral matrix inequality [11]). For any symmetric positive definite matrix $M>0$, scalar $\gamma>0$ and vector function $\omega:[0, \gamma] \rightarrow \mathbb{R}^{n}$ such that the integrations concerned are well defined, the following inequality holds

$$
\left(\int_{0}^{\gamma} \omega(s) d s\right)^{T} M\left(\int_{0}^{\gamma} \omega(s) d s\right) \leq \gamma\left(\int_{0}^{\gamma} \omega^{T}(s) M \omega(s) d s\right)
$$

\section{Main Results}

In this section, we give a design of memoryless guaranteed feedback cost control for uncertain switched recurrent neural networks (1). Let us set

$$
\begin{aligned}
& W_{11}=-P A_{i}^{T}-A_{i} P-2 \alpha P+0.25 B_{i} R B_{i}^{T}+2 \epsilon_{1} E_{a}^{T} E_{a}+2 \epsilon_{1}^{-1} P H_{a}^{T} H_{a} P \\
& +2 \epsilon_{2}^{-1} P F H_{w_{0}}^{T} H_{w_{0}} F P, W_{12}=P+A_{i} P-0.5 B_{i} B_{i}^{T}, \\
& W_{13}=e^{-2 \alpha h_{0}} H_{0}+0.5 B_{i} B_{i}^{T}+A_{i} P, W_{14}=e^{-2 \alpha h_{1}} H_{1}+0.5 B_{i} B_{i}^{T}+A_{i} P, \\
& W_{15}=P+0.5 B_{i} B_{i}^{T}+A_{i} P, W_{22}=\sum_{j=0}^{1} W_{j i} D_{j} W_{j i}^{T}+\sum_{i=0}^{1} h_{i}^{2} H_{i}-2 P-B_{i} B_{i}^{T} \\
& +\epsilon_{1} E_{a}^{T} E_{a}+\epsilon_{2} E_{w_{0 \mathrm{i}}}^{T} E_{w_{0 \mathrm{i}}}+\epsilon_{3} E_{w_{1 i}}^{T} E_{w_{1 \mathrm{i}}}, W_{23}=P, W_{24}=P, W_{25}=P, \\
& W_{33}=-e^{-2 \alpha h_{0}} H_{0}+\sum_{j=0}^{1} W_{j i} D_{j} W_{j i}^{T}+\epsilon_{1} E_{a}^{T} E_{a}+\epsilon_{2} E_{w_{0}}^{T} E_{w_{0}}+\epsilon_{3} E_{w_{1}}^{T} E_{w_{1}}, \\
& W_{44}=\sum_{j=0}^{1} W_{j i} D_{j} W_{j i}^{T}-e^{-2 \alpha h_{1}} H_{1}+\epsilon_{1} E_{a}^{T} E_{a}+\epsilon_{2} E_{w_{0}}^{T} E_{w_{0}}+\epsilon_{3} E_{w_{1 i}}^{T} E_{w_{1}}, \\
& W_{55}=W_{0} D_{0} W_{0}^{T}+2 \epsilon_{3}^{-1} P E H_{w_{1}}^{T} H_{w_{1}} E P+\epsilon_{1} E_{a}^{T} E_{a}+\epsilon_{2} E_{w_{0 i}}^{T} E_{w_{0}}+\epsilon_{3} E_{w_{1}}^{T} E_{w_{1}}, \\
& E=\operatorname{diag}\left\{e_{i}, i=1, \ldots, n\right\}, F=\operatorname{diag}_{i}\{i=1, \ldots, n\}, \lambda_{1}=\lambda_{\min }\left(P^{-1}\right), \\
& \lambda_{2}=\lambda_{\max }\left(P^{-1}\right)+h_{1}^{2} \lambda_{\max }\left[P^{-1}\left(\sum_{i=0}^{1} H_{i}\right) P^{-1}\right] .
\end{aligned}
$$

Theorem 1. Consider control system (1) and the cost function (5). If there exist symmetric positive definite matrices $P, H_{0}, H_{1}$, diagonal positive definite matrices $D_{i}, i=0,1$, and $\epsilon_{i}>0, i=1,2,3$ satisfying the following $L M I_{S}$

$$
\mathcal{E}_{i}=\left[\begin{array}{ccccc}
W_{11} & W_{12} & W_{13} & W_{14} & W_{15} \\
* & W_{22} & W_{23} & W_{24} & W_{25} \\
* & * & W_{33} & 0 & 0 \\
* & * & * & W_{44} & 0 \\
* & * & * & * & W_{55}
\end{array}\right]<0
$$




$$
\begin{aligned}
& \mathcal{S}_{1 i}=\left[\begin{array}{ccc}
-P A_{i}-A_{i}^{T} P-\sum_{j=0}^{1} e^{-2 \alpha h_{j}} H_{j} & 2 P F & P Q_{1} \\
* & -D_{0} & 0 \\
* & * & -Q_{1}^{-1}
\end{array}\right]<0, \\
& \mathcal{S}_{2 i}=\left[\begin{array}{ccc}
W_{1 i} D_{1} W_{1 i}^{T} & 2 P E & P Q_{2} \\
* & -D_{1} & 0 \\
* & * & -Q_{2}^{-1}
\end{array}\right]<0
\end{aligned}
$$

then

$$
u(t)=-\frac{1}{2} B_{i}^{T} P^{-1} x(t), \quad t \geq 0 .
$$

is a guaranteed cost control and the guaranteed cost value is given by

$$
J^{*}=\lambda_{2}\|\phi\|^{2}
$$

Moreover, the solution $x(t, \phi)$ of the system satisfies

$$
\|x(t, \phi)\| \leq \sqrt{\frac{\lambda_{2}}{\lambda_{1}}} e^{-\alpha t}\|\phi\|, \quad \forall t \geq 0
$$

Proof. Let $Y=P^{-1}, y(t)=Y x(t)$. Using the feedback control (11) we consider the following Lyapunov-Krasovskii functional

$$
\begin{gathered}
V\left(t, x_{t}\right)=\sum_{i=1}^{3} V_{i}\left(t, x_{t}\right), \\
V_{1}=x^{T}(t) Y x(t), \\
V_{2}=h_{0} \int_{-h_{0}}^{0} \int_{t+s}^{t} e^{2 \alpha(\tau-t)} \dot{x}^{T}(\tau) Y H_{0} Y \dot{x}(\tau) d \tau d s, \\
V_{3}=h_{1} \int_{-h_{1}}^{0} \int_{t+s}^{t} e^{2 \alpha(\tau-t)} \dot{x}^{T}(\tau) Y H_{1} Y \dot{x}(\tau) d \tau d s .
\end{gathered}
$$

It easy to check that

$$
\lambda_{1}\|x(t)\|^{2} \leq V\left(t, x_{t}\right) \leq \lambda_{2}\left\|x_{t}\right\|^{2}, \quad \forall t \geq 0
$$


Taking the derivative of $V_{i}, i=1,2,3$ we have

$$
\begin{aligned}
\dot{V}_{1}= & 2 x^{T}(t) Y \dot{x}(t) \\
= & y^{T}(t)\left[-P\left(A_{i}+E_{a} F_{a}(t) H_{a}\right)^{T}-\left(A_{i}+E_{a} F_{a}(t) H_{a}\right) P\right] y(t) \\
& -y^{T}(t) B_{i} B_{i}^{T} y(t) \\
& +2 y^{T}(t)\left(W_{0 i}+E_{w_{0}} F_{w_{0}}(t) H_{w_{0}}\right) f(.) y(t)+2 y^{T}(t)\left(W_{1 i}\right. \\
& \left.+E_{w_{1}} F_{w_{1}}(t) H_{w_{1}}\right) g(.) y(t), \\
\dot{V}_{2}= & h_{0}^{2} \dot{y}^{T}(t) H_{0} \dot{y}(t)-h_{1} e^{-2 \alpha h_{0}} \int_{t-h_{0}}^{t} \dot{x}^{T}(s) H_{0} \dot{x}(s) d s-2 \alpha V_{2}, \\
\dot{V}_{3}= & h_{1}^{2} \dot{y}^{T}(t) H_{1} \dot{y}(t)-h_{1} e^{-2 \alpha h_{1}} \int_{t-h_{1}}^{t} \dot{y}^{T}(s) H_{1} \dot{y}(s) d s-2 \alpha V_{3} .
\end{aligned}
$$

Applying Proposition 2 and the Leibniz - Newton formula

$$
\int_{s}^{t} \dot{y}(\tau) d \tau=y(t)-y(s),
$$

we have for $j=1,2, i=0,1$ :

$$
\begin{aligned}
-h_{i} \int_{t-h_{\mathrm{i}}}^{t} \dot{y}^{T}(s) H_{j} \dot{y}(s) d s & \leq-\left[\int_{t-h_{\mathrm{i}}}^{t} \dot{y}(s) d s\right]^{T} H_{j}\left[\int_{t-h_{\mathrm{i}}}^{t} \dot{y}(s) d s\right] \\
& \leq-[y(t)-y(t-h(t))]^{T} H_{j}[y(t)-y(t-h(t))] \\
& =-y^{T}(t) H_{i} y(t)+2 x^{T}(t) H_{j} y(t-h(t)) \\
& -y^{T}\left(t-h_{i}\right) H_{j} y\left(t-h_{i}\right) .
\end{aligned}
$$

Then, we have

$$
\begin{aligned}
\dot{V}(.)+2 \alpha V(.) & \leq y^{T}(t)\left[-P\left(A_{i}+E_{a} F_{a}(t) H_{a}\right)^{T}-\left(A_{i}+E_{a} F_{a}(t) H_{a}\right) P\right] y(t) \\
& -y^{T}(t) B_{i} B_{i}^{T} y(t)+2 y^{T}(t)\left(W_{0 i}+E_{w_{0 \mathrm{i}}} F_{w_{0 \mathrm{i}}}(t) H_{w_{0 \mathrm{i}}}\right) f(.) \\
& +2 y^{T}(t)\left(W_{1 i}+E_{w_{1 \mathrm{i}}} F_{w_{1 \mathrm{i}}}(t) H_{w_{1 \mathrm{i}}}\right) g(.)+2 \alpha\langle P y(t), y(t)\rangle \\
& +\dot{y}^{T}(t)\left(\sum_{i=0}^{1} h_{i}^{2} H_{i}\right) \dot{y}(t)-\sum_{i=0}^{1} e^{-2 \alpha h_{\mathrm{i}}} y^{T}\left(t-h_{i}\right) G_{i} y\left(t-h_{i}\right) \\
& -e^{-2 \alpha h_{0}}\left[y(t)-y\left(t-h_{0}\right)\right]^{T} H_{0}\left[y(t)-y\left(t-h_{0}\right)\right] \\
& -e^{-2 \alpha h_{1}}\left[y(t)-y\left(t-h_{1}\right)\right]^{T} H_{1}\left[y(t)-y\left(t-h_{1}\right)\right] \\
& =\zeta^{T}(t) \mathcal{E}_{i} \zeta(t)+y^{T}(t) S_{1 i} y(t)+y^{T}(t-h(t)) S_{2 i} y(t-h(t)) \\
& -f^{0}(t, x(t), x(t-h(t)), u(t)),
\end{aligned}
$$


where $\zeta(t)=\left[y(t), \dot{y}(t), y\left(t-h_{0}\right), y\left(t-h_{1}\right), y(t-h(t))\right]$. Integrating both sides of (14) from 0 to $t$, we obtain

$$
V\left(t, x_{t}\right) \leq V(\phi) e^{-2 \alpha t}, \quad \forall t \geq 0 .
$$

Furthermore, taking condition (12) into account, we have

$$
\lambda_{1}\|x(t, \phi)\|^{2} \leq V\left(x_{t}\right) \leq V(\phi) e^{-2 \alpha t} \leq \lambda_{2} e^{-2 \alpha t}\|\phi\|^{2},
$$

then

$$
\|x(t, \phi)\| \leq \sqrt{\frac{\lambda_{2}}{\lambda_{1}}} e^{-\alpha t}\|\phi\|, \quad t \geq 0
$$

which concludes the exponential stability of the closed-loop system (7). To prove the optimal level of the cost function (5), we derive from (14) and (8) (10) that

$$
\dot{V}\left(t, z_{t}\right) \leq-f^{0}(t, x(t), x(t-h(t)), u(t)), \quad t \geq 0 .
$$

Integrating both sides of (15) from 0 to $t$ leads to

$$
\int_{0}^{t} f^{0}(t, x(t), x(t-h(t)), u(t)) d t \leq V\left(0, z_{0}\right)-V\left(t, z_{t}\right) \leq V\left(0, z_{0}\right),
$$

dute to $V\left(t, z_{t}\right) \geq 0$. Hence, letting $t \rightarrow+\infty$, we have

$$
J=\int_{0}^{\infty} f^{0}(t, x(t), x(t-h(t)), u(t)) d t \leq V\left(0, z_{0}\right) \leq \lambda_{2}\|\phi\|^{2}=J^{*} .
$$

This completes the proof of the theorem.

Remark 1. Note that $h(t)$ is non-differentiable and interval time-varying delay, therefore, the stability criteria proposed in $[2,3,5,7-10]$ are not applicable to this system.

\section{Conclusion}

In this paper, the problem of guaranteed cost control for uncertain switched recurrent neural networks with interval nondifferentiable time-varying delay has been studied. A nonlinear quadratic cost function is considered as a performance measure for the closed-loop system. The stabilizing controllers to be designed must satisfy some exponential stability constraints on the closed-loop poles. By constructing a set of time-varying Lyapunov-Krasovskii functional 
combined with Newton-Leibniz formula, a memoryless state feedback guaranteed cost controller design has been presented and sufficient conditions for the existence of the guaranteed cost state-feedback for the system have been derived in terms of LMIs.

\section{Acknowledgements}

This work was supported by the Thailand Research Fund Grant, the Commission for Higher Education and Faculty of Science, Maejo University, Thailand. The authors thank anonymous reviewers for valuable comments and suggestions, which allowed us to improve the paper.

\section{References}

[1] Hopfield J.J., Neural networks and physical systems with emergent collective computational abilities, Proc. Natl. Acad. Sci. USA, 79(1982), 2554-2558.

[2] K. Ratchagit, Asymptotic stability of delay-difference system of Hopfield neural networks via matrix inequalities and application, International Journal of Neural Systems, 17(2007), 425-430. DOI: 10.1142/S0129065707001263

[3] Park J.H., On global stability criterion for neural networks with discrete and distributed delays, Chaos, Solitons and Fractals, 30(2006), 897-902.

[4] Kreangkri Ratchagit, Stability analysis of linear systems with time delays, International Journal of Pure and Applied Mathematics, Vol. 76, No. 1, $2012,21-28$.

[5] Fridman E., Orlov Y., Exponential stability of linear distributed parameter systems with time-varying delays. Automatica, 45(2009), 194-201.

[6] Kreangkri Ratchagit, Stability criteria of LPD system with time-varying delay, International Journal of Pure and Applied Mathematics, Vol. 78 No. $6,2012,857-866$.

[7] K. Ratchagit and V.N. Phat, Stability criterion for discrete-time systems, Journal of Inequalities and Applications, 2010, 2010:201459 doi:10.1155/2010/201459. 
[8] V.N. Phat, Y. Khongtham, and K. Ratchagit, LMI approach to exponential stability of linear systems with interval time-varying delays. Linear Algebra and its Applications, Vol. 436, 2012, 243-251. DOI: 10.1016/j.laa.2011.07.016

[9] Xu S., Lam J., A survey of linear matrix inequality techniques in stability analysis of delay systems. Int. J. Syst. Sci., 39(2008), 12, 1095-1113.

[10] K. Ratchagit, V.N. Phat, Stability and stabilization of switched linear discrete-time systems with interval time-varying delay, Nonlinear Anal. Hybrid Syst., Vol. 5, 2011, 605-612. DOI: 10.1016/j.nahs.2011.05.006

[11] R. P. Agarwal, Difference Equations and Inequalities, Second Edition, Marcel Dekker, New York, 2000. 
